\title{
Regulation of YKL-40 expression by corticosteroids: effect on pro-inflammatory macrophages in vitro and its modulation in COPD in vivo
}

L. I. Z. Kunz ${ }^{1 * \dagger}$, E. F. A. van't Wout ${ }^{1 \dagger}$, A. van Schadewijk1', D. S. Postma², H. A. M. Kerstjens' ${ }^{2}$, P. J. Sterk ${ }^{3}$ and P. S. Hiemstra ${ }^{1}$

\begin{abstract}
Background: Macrophages constitute a heterogeneous cell population with pro- (MФ1) and anti-inflammatory (MФ2) cells. The soluble chitinase-like-protein YKL-40 is expressed in macrophages and various other cell types, and has been linked to a variety of inflammatory diseases, including COPD. Dexamethasone strongly reduces YKL-40 expression in peripheral blood mononuclear cells (PBMC) in vitro. We hypothesized that: a) YKL-40 is differentially expressed by $M \Phi 1$ and $M \Phi 2, b)$ is decreased by corticosteroids and c) that long-term treatment with inhaled corticosteroids (ICS) affects YKL-40 levels in serum and sputum of COPD patients.
\end{abstract}

Methods: Monocytes of healthy subjects were cultured in vitro for 7 days with either GM-CSF or M-CSF (for MФ1 and MФ2, respectively) and stimulated for $24 \mathrm{~h}$ with LPS, TNFa, or oncostatin M (OSM). MФ1 and MФ2 differentiation was assessed by measuring secretion of IL-12p40 and IL-10, respectively. YKL-40 expression in macrophages was measured by quantitative RT-PCR (qPCR) and ELISA; serum and sputum YKL-40 levels were analyzed by ELISA.

Results: Pro-inflammatory MФ1 cells secreted significantly more YKL-40 than MФ2, which was independent of stimulation with LPS, TNFa or OSM $(p<0.001)$ and confirmed by GPCR. Dexamethasone dose-dependently and significantly inhibited YKL-40 protein and mRNA levels in MФ1. Serum YKL-40 levels of COPD patients were significantly higher than sputum YKL-40 levels but were not significantly changed by ICS treatment.

Conclusions: YKL-40 secretion from MФ1 cells is higher than from MФ2 cells and is unaffected by further stimulation with pro-inflammatory agents. Furthermore, YKL-40 release from cultured monocyte-derived macrophages is inhibited by dexamethasone especially in MD1, but ICS treatment did not change YKL-40 serum and sputum levels in COPD. These results indicate that YKL-40 expression could be used as a marker for MФ1 macrophages in vitro, but not for monitoring the effect of ICS in COPD.

Trial registration: ClinicalTrials.gov, registration number: NCT00158847

\footnotetext{
* Correspondence: L.I.Z.Kunz@lumc.nl

${ }^{\dagger}$ Equal contributors

'Department of Pulmonology, Leiden University Medical Center, Albinusdreef

2, NL-2333ZA, Leiden, The Netherlands

Full list of author information is available at the end of the article
} 


\section{Background}

YKL-40 (or chitinase 3-like-1 [CHI3L1], breast regression protein [BRP]-39 or human cartilage glycoprotein39 [HC gp-39]) is a chitinase-like protein which is found in humans [1]. It is expressed by various cell types, including neutrophils and macrophages [2-4], while macrophages have been identified as its main cellular source $[2,5]$. Monocytes do not express YKL-40, and YKL-40 expression appears to be associated with later stages of macrophage differentiation $[2,6]$. Although its biological function is largely unknown, YKL-40 has been suggested to play a major role in a variety of processes, including epithelial-mesenchymal transition, migration and proliferation of (malignant) cells, angiogenesis, tissue remodeling and inflammation [7-9]. Furthermore, YKL-40 has been implicated in several acute and chronic inflammatory diseases, including asthma and COPD [10, 11]. Several studies have shown that YKL-40 levels are higher in sputum and serum of COPD patients compared to asymptomatic smokers [12, 13].

These data suggest that macrophages may also be a major source of YKL-40 in inflammatory lung diseases such as COPD, a disease in which macrophages play an important role [14]. It is however unknown whether YKL40 expression is restricted to a subset of macrophages. This is a relevant question, since macrophages constitute heterogeneous cell populations with various functions. Pro-inflammatory, or classically activated macrophages (type I, MФ1), show pro-inflammatory properties by secreting pro-inflammatory cytokines such as interleukin (IL)-12, have antigen presenting capacity and promote Th1 immunity. In contrast, anti-inflammatory, or alternatively activated macrophages (type II, MФ2), demonstrate anti-inflammatory characteristics with expression and secretion of anti-inflammatory cytokines, such as IL-10 and promote development of regulatory $\mathrm{T}$ cells [15]. Furthermore, Di Rosa et al. observed higher expression of YKL-40 in monocyte-derived MФ1 [16].

Dexamethasone strongly suppresses YKL-40 expression in cultured monocyte-derived macrophages [17]. In line with these findings, rheumatoid arthritis patients treated with methotrexate and prednisolone had lower serum YKL-40 concentrations compared to patients treated without prednisolone [18]. However, it is unknown if YKL-40 levels are influenced by treatment with inhaled corticosteroids (ICS) in COPD and could therefore be used to monitor treatment response. Furthermore, it is not known whether modulation by steroids is a direct genomic anti-inflammatory effect or due to e.g. epigenetic mechanisms [19-22].

Based on these observations we hypothesized that YKL40 expression is directed by macrophage polarization, with a higher expression in monocyte-derived $M \Phi 1$ compared to МФ2. Furthermore, we hypothesized that steroids decrease YKL-40 secretion and expression in $M Ф 1$ and that YKL-40 in serum and sputum of COPD patients is decreased by treatment with ICS. The results from this study show that monocyte-derived MФ1 secrete and express more YKL-40 than MФ2. YKL-40 expression by cultured MФ1 cells is steroid sensitive as its production is decreased by dexamethasone. Finally, YKL-40 levels in sputum and serum do not differ between COPD patients treated for 30 months with ICS or placebo.

\section{Methods \\ Cell culture}

Monocytes were isolated from buffy coats of healthy blood donors (Sanquin Blood Bank, Leiden, The Netherlands) using anti-CD14 microbeads (Miltenyi Biotec, Auburn, CA, USA) according to the manufacturer's protocol. $М Ф 1$ and MФ2 were derived as described previously [23, 24]. Briefly, cells were cultured for six days in RPMI 1640 medium in 48 well plates (Invitrogen, Life Technologies, Bleiswijk, The Netherlands) containing $10 \%$ fetal calf serum (FCS, Invitrogen), $2 \mathrm{mM}$ L-glutamine, 100U/ml penicillin and $100 \mu \mathrm{g} / \mathrm{ml}$ streptomycin (all Bio Whittaker, Walkersville, MD, USA) at $37{ }^{\circ} \mathrm{C}$ in $5 \% \mathrm{CO}_{2}$ atmosphere with either GM-CSF ( $5 \mathrm{ng} / \mathrm{ml}$, Invitrogen) or M-CSF (50 ng/ml, R\&D Systems, Minneapolis, MN, USA) to obtain $M \Phi 1$ and $M \Phi 2$, respectively [13, 14]. Differentiated macrophages were stimulated with the pro-inflammatory stimuli lipopolysaccharide (LPS, from Pseudomonas aeruginosa, $100 \mathrm{ng} / \mathrm{ml}$, Sigma-Aldrich, St. Louis, MO, USA), TNF- $\alpha$ (10 ng/ml, Peprotech, Rocky Hill, NJ, USA) or oncostatin M (OSM, $100 \mathrm{ng} / \mathrm{ml}$, R\&D Systems) for $24 \mathrm{~h}$. Dexamethasone (0.1, 0.3 and $1 \mathrm{nM}$, Sigma) was added during differentiation at day 0,3 and/or day 7 . The demethylating agent 5-AZA-2'-deoxycytidine (5-AZA, 0.1, 1 and $10 \mu \mathrm{M}$, Sigma) was added during differentiation. Every day $100 \mu \mathrm{l}$ per well was removed and replaced by fresh medium containing growth factors and 5-AZA.

\section{GLUCOLD study}

Serum and sputum supernatants were obtained from patients with moderate to severe COPD who participated in the GLUCOLD (Groningen and Leiden Universities Corticosteroids in Obstructive Lung Disease) study [25]. Patients were steroid-naive at baseline and were subsequently randomized to one of four inhaled treatments, all twice daily: 6- or 30-month fluticasone propionate dry-powder inhaler (500 $\mu \mathrm{g}$, group 1 and 2, respectively), 30 -month fluticasone with salmeterol $(500 / 50 \mu \mathrm{g}$, group 3) or 30-month placebo (group 4). In this mechanistic study, we only used data from the compliant patients ( $\geq 70 \%$ of the prescribed dose of treatment) of groups 2 and 3 combined to increase power, and group 4 . At baseline and after 30 months of treatment, serum and sputum samples were collected. Sputum induction and processing 
were performed as previously described $[26,27]$. Cell free supernatants of serum and sputum were stored at $-80{ }^{\circ} \mathrm{C}$. The ethics committees of Leiden University Medical Center and University Medical Center Groningen approved the study and all patients provided written informed consent.

\section{Enzyme-linked Immunosorbent Assay (ELISA)}

Commercially available ELISA kits were used to detect IL-12p40 (IL-12/IL-23p40, R\&D Systems; sensitivity
$62.5 \mathrm{pg} / \mathrm{ml}$ ) and IL-10 (Sanquin, Amsterdam, The Netherlands; sensitivity $4.1 \mathrm{pg} / \mathrm{ml}$ ), to confirm that the monocytes were adequately differentiated towards MФ1 and MФ2, respectively (Fig. 1a). YKL-40 ELISA (R\&D Systems; sensitivity $16 \mathrm{pg} / \mathrm{ml}$ [10]) was performed on cell culture supernatant, serum and sputum supernatant. The absorbance was measured at $450 \mathrm{~nm}$ using a Microplate reader (model 680; Bio-Rad, Hercules, CA, USA) and Microplate Manager software (version 5.2.1, Bio-Rad).

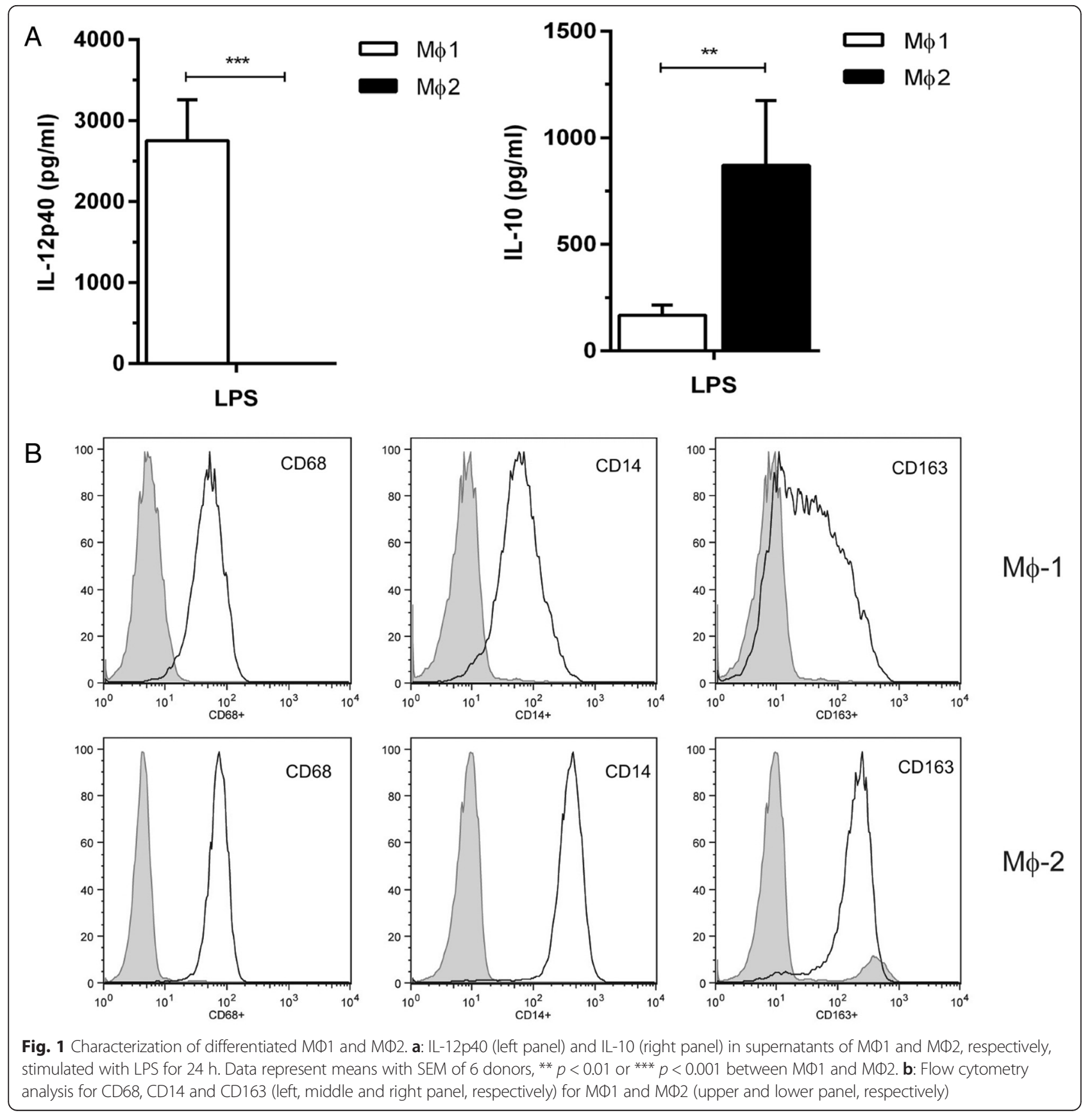




\section{Flow cytometry}

Cell surface markers were assessed by standard flow cytometry using a FACS Calibur cytometer (Becton and Dickinson, La Jolla, CA, USA) and CellQuest Pro software after staining with specific APC-conjugated CD163 and CD14 (both BD Biosciences/Pharmingen (Temse, Belgium), FITC-conjugated CD68 (eBioscience, Vienna, Austria) and/or goat-anti-human YKL-40 (R\&D systems) detected by Alexa Fluor $594 \mathrm{~F}(\mathrm{ab})_{2}$ fragment of goat anti-mouse (Invitrogen) as secondary antibody. Cells were incubated with the antibodies for $30 \mathrm{~min}$ on ice in PBS containing $0.5 \%$ BSA (w/v) and $0.2 \%$ sodium azide (w/v) (both Sigma). After fixation with Cytofix/Cytoperm buffer, intracellular staining was performed in Perm/Wash buffer (both BD Biosciences). Flow cytometric analysis confirmed that the differentiated monocytes were $\mathrm{CD} 14^{+}$ and $\mathrm{CD}_{68}^{+}$, and that the majority of $\mathrm{M \Phi} 2$ were $\mathrm{CD} 163^{+}$ (Fig. 1b). Therefore, we concluded that the monocytes were properly differentiated into MФ1 and MФ2 cells.

\section{Immunofluorescence staining}

YKL-40 and CD68 expression in sputum cytospins was demonstrated using immunofluorescence. Cytospins were fixed in $4 \%$ formaldehyde in PBS for $30 \mathrm{~min}$, followed by antigen retrieval in citrate solution pH6.0 (DAKO, Glostrup, Denmark) for $30 \mathrm{~min}$ and cooled on ice. The primary antibodies goat-anti-human YKL-40 (R\&D systems, dilution 1:25) and mouse-anti-human CD68 (clone PG-M1, DAKO, dilution 1:50), diluted in PBS/1 \% BSA $(\mathrm{w} / \mathrm{v})$ were incubated together overnight. Alexa Fluor568 donkey-anti-goat and Alexa Fluor 488 donkey-anti-mouse (Invitrogen, Eugene, Oregon, USA, dilution of both 1:200) were incubated in a dark environment for $30 \mathrm{~min}$. The cytospins were covered with Vectashield with DAPI (Vector Laboratories, Inc. Burlingame, CA, USA). Photographs were taken with a confocal microscope.

\section{Quantitative reverse-transcriptase polymerase chain reaction (qPCR)}

RNA was isolated using Qiagen RNeasy mini kit (Qiagen, Venlo, The Netherlands) and cDNA was synthesized in equal amounts per experiment. Quantitative reversetranscriptase polymerase chain reaction (qPCR) was performed with the primers for YKL-40 and LL-37 as presented in Table 1. QPCR was performed on the iCycler PCR device using iQ SYBR Green Supermix (Bio-Rad) for 40 cycles at $58{ }^{\circ} \mathrm{C}$. Relative mRNA concentrations of ACTB and ATP5B (GeNorm, PrimerDesign
Ltd., Southampton, UK) were used as housekeeping genes for human genes.

\section{Statistical analysis}

Differences within one cell type and between cell types were analyzed by one-way and two-way ANOVA, respectively. Paired and unpaired tests were used for evaluating YKL-40 levels in serum and sputum within and between treatments, respectively, using only data from subjects with availability of samples from both baseline and 30 months. Statistical analysis was performed with SPSS 22.0 software (SPSS Inc., Chicago, IL). Data are presented as means with standard error of the mean (SEM) for in vitro experiments and means with standard deviations (SD) for serum and sputum samples. Differences at pvalues $\leq 0.05$ were inferred as statistically significant.

\section{Results}

MФ1 produce more YKL-40 compared to MФ2

Secretion of YKL-40 by MФ1 was markedly higher compared to MФ2 $(p<0.001)$ and not further increased by stimulation for $24 \mathrm{~h}$ with $100 \mathrm{ng} / \mathrm{ml}$ LPS compared to medium ( $p<0.001$; Fig. 2a, left panel). qPCR confirmed these results, i.e. MФ1 express more YKL-40 than MФ2 with and without LPS stimulation (10-fold higher in MФ1 vs $М Ф 2, p<0.001$; Fig. 2a, right panel), with a trend towards more YKL-40 expression in LPS-stimulated MФ1 $(p<0.1)$. Flow cytometry analysis also showed that MФ1 cells express more YKL-40 compared to MФ2 (Fig. 2b). In addition, MФ1 secreted more YKL-40 compared to MФ2 which was irrespective of further stimulation with TNF- $\alpha$, and OSM for 24 or $48 \mathrm{~h}$, respectively (all $p<0.001$, Fig. 2c). Immunofluorescence staining showed that only a minority of macrophages in sputum of COPD patients was positive for YKL-40 as shown by costaining with CD68 (Fig. 2d).

\section{YKL-40 expression is inhibited by dexamethasone}

To investigate the effect of steroids on macrophage YKL-40 expression, we first assessed its effect on expression of the MФ1 and MФ2 markers IL-12p40 and IL-10. Whereas dexamethasone did not affect IL-10 secretion, it inhibited IL-12p40 secretion, especially when added at day 0 (Fig. 3a and b, respectively). In line with these findings, dexamethasone also dose-dependently inhibited YKL-40 expression and secretion mainly in MФ1 (Fig. 4a and $b$, respectively). Again this effect was most pronounced when dexamethasone was added from the start

Table 1 Primer pairs used for quantitative reverse-transcriptase polymerase chain reaction (qPCR)

\begin{tabular}{lll}
\hline & Forward & Reverse \\
\hline YKL-40 (CHI3L1)(154 base pairs) & CTG TGG GGA TAG TGA GGC AT & CTT GCC AAA ATG GTG TCC TT \\
LL-37 (CAMP)(249 base pairs) & TCA TTG CCC AGG TCC TCA G & TCC CCA TAC ACC GCT TCA C \\
\hline
\end{tabular}




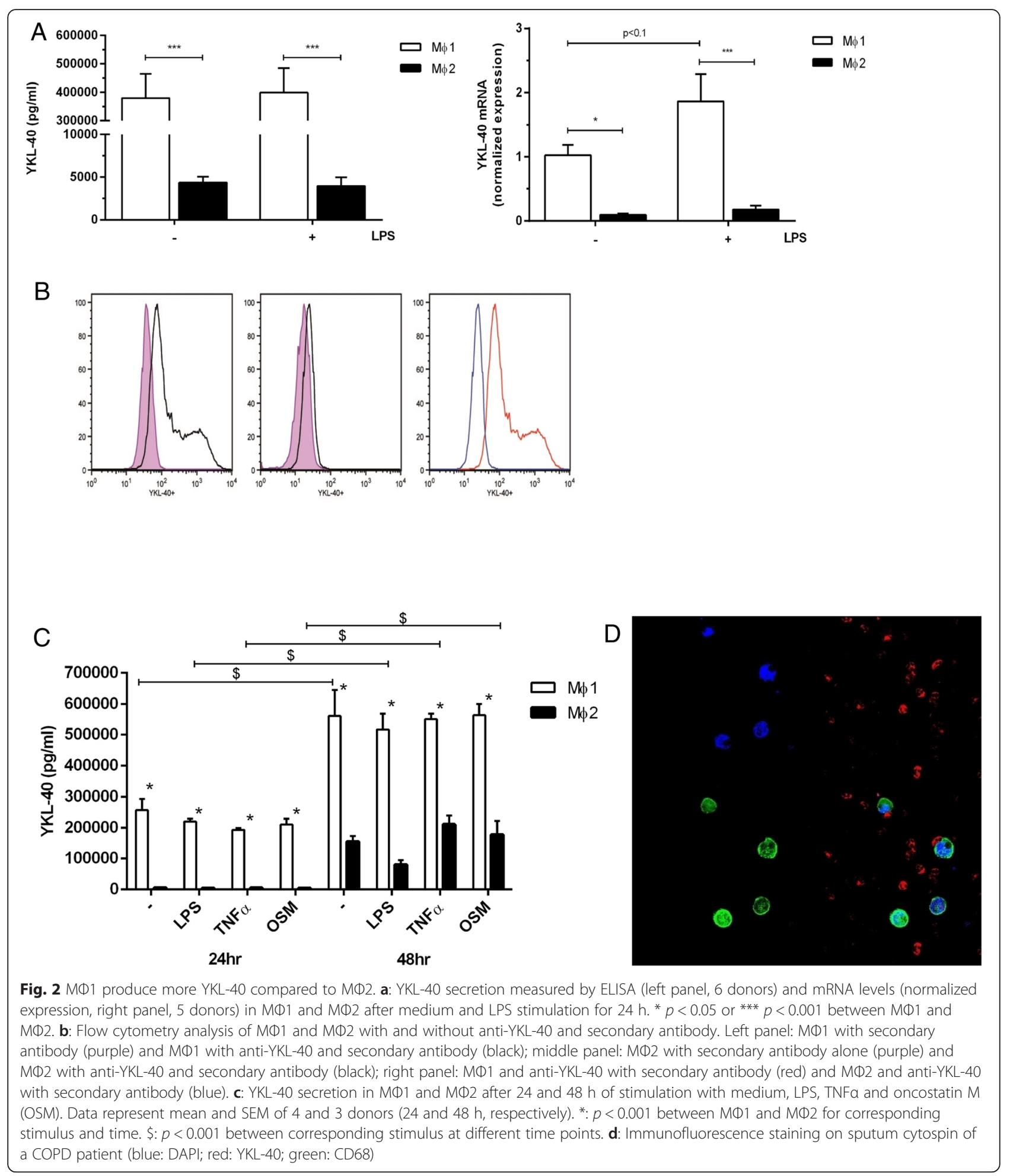

of the differentiation at day 0 (Fig. $4 \mathrm{a}$ and b, left panel). Adding dexamethasone at later time points (day 3 or day 7) showed a trend for lower YKL-40 expression and secretion (Fig. 4a and b, middle and right panel, respectively), but failed to reach significance.
We have previously demonstrated that the human cathelicidin antimicrobial peptide hCAP/LL-37 directs macrophage differentiation towards MФ1 cells [28]. In the course of the latter studies we also noted that hCAP18/LL-37 is preferentially expressed in MФ1 cells 

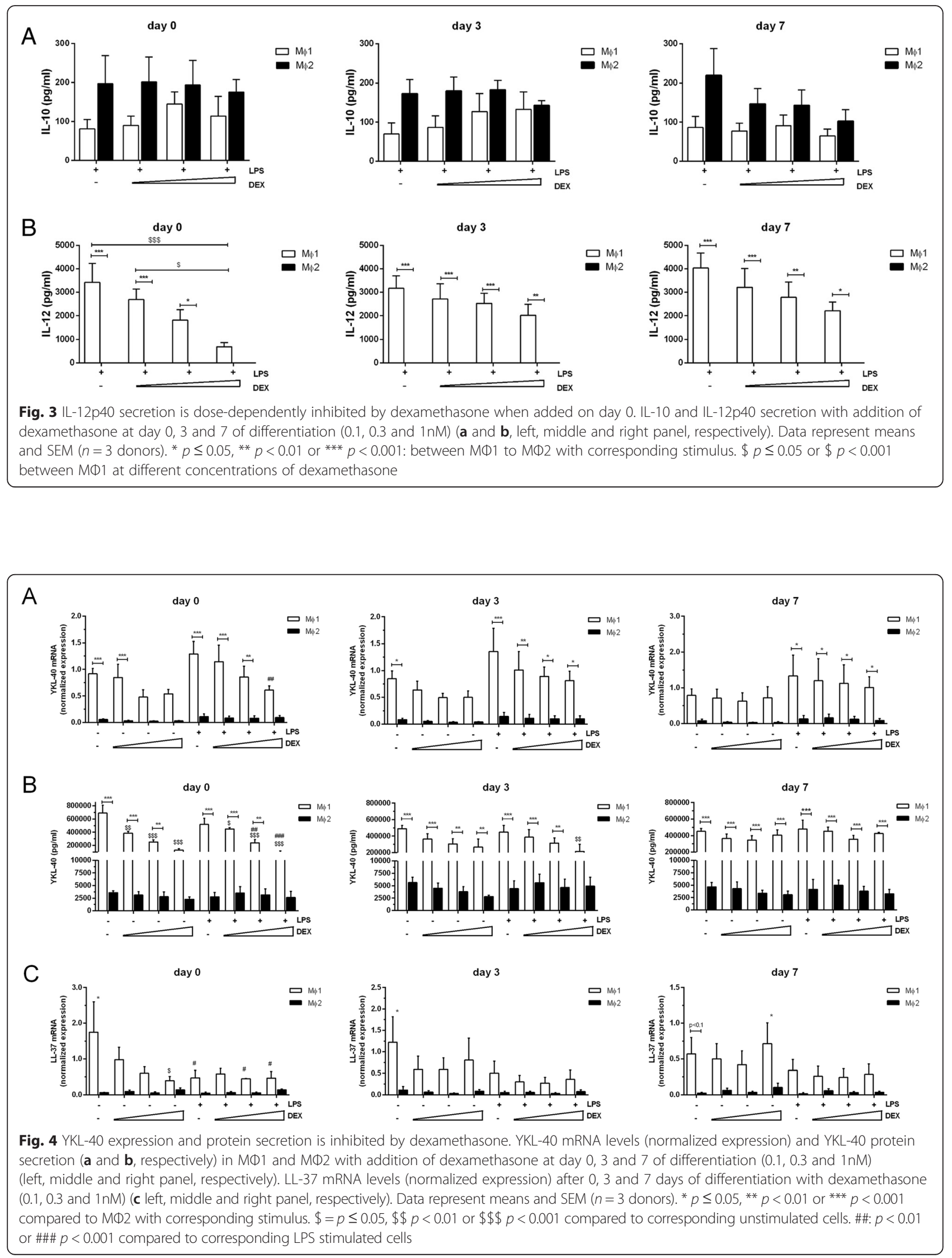
(unpublished results). In the present study, we therefore used LL-37 expression for a comparison with YKL-40 expression. In line with the findings for IL-12p40 and YKL40, the expression of LL-37 was also dose-dependently reduced by increasing doses of dexamethasone when added on day 0 , but not on day 3 or day 7 (Fig. 4c).

Collectively these data indicate that dexamethasone inhibits YKL-40 expression, but that this effect is most likely explained by a more generic effect on $M \Phi 1$ development and is not selective for YKL-40 expression.

\section{YKL-40 expression is not affected by the demethylating agent 5-AZA}

Epigenetic mechanisms may contribute to CHI3L1 expression. This is supported by the finding of a singlenucleotide polymorphism (SNP) localized near the CpG island in the promoter region of the CHI3L1 gene, which is associated with YKL-40 expression $[19,20]$. To evaluate whether YKL-40 expression in monocyte-derived macrophages is influenced by the methylation status of the CHI3L1 gene, macrophages were generated in the presence of the demethylating agent 5-AZA. We first observed that 5-AZA inhibited IL-12p40 secretion, and to a smaller extent also IL-10 secretion (Fig. 5a and b, respectively). In contrast, YKL-40 protein secretion and expression was not significantly affected by 5AZA, although it needs to be noted that the highest concentration resulted in cell toxicity (Fig. $5 \mathrm{c}$ and d, respectively).

\section{YKL-40 levels in serum and sputum of COPD patients are} not changed by treatment with inhaled corticosteroids We next used samples of the GLUCOLD study to investigate the effects of ICS treatment on serum and sputum YKL-40 levels in COPD patients. Baseline characteristics between the group of moderate-to-severe COPD patients treated with ICS and placebo were not significantly different as shown in Table 2. Of the 75 compliant patients, 70 serum samples and 59 induced sputum samples at baseline were available and suitable for analysis. Serum YKL-40 levels at baseline were significantly higher compared to sputum levels at baseline (respectively median $71 \mathrm{ng} / \mathrm{ml}$ versus $29 \mathrm{ng} / \mathrm{ml}, p<0.001$ ). ICS treatment did not significantly change YKL-40 levels in serum and sputum compared to placebo (both $p>0.05$; Fig. 6).
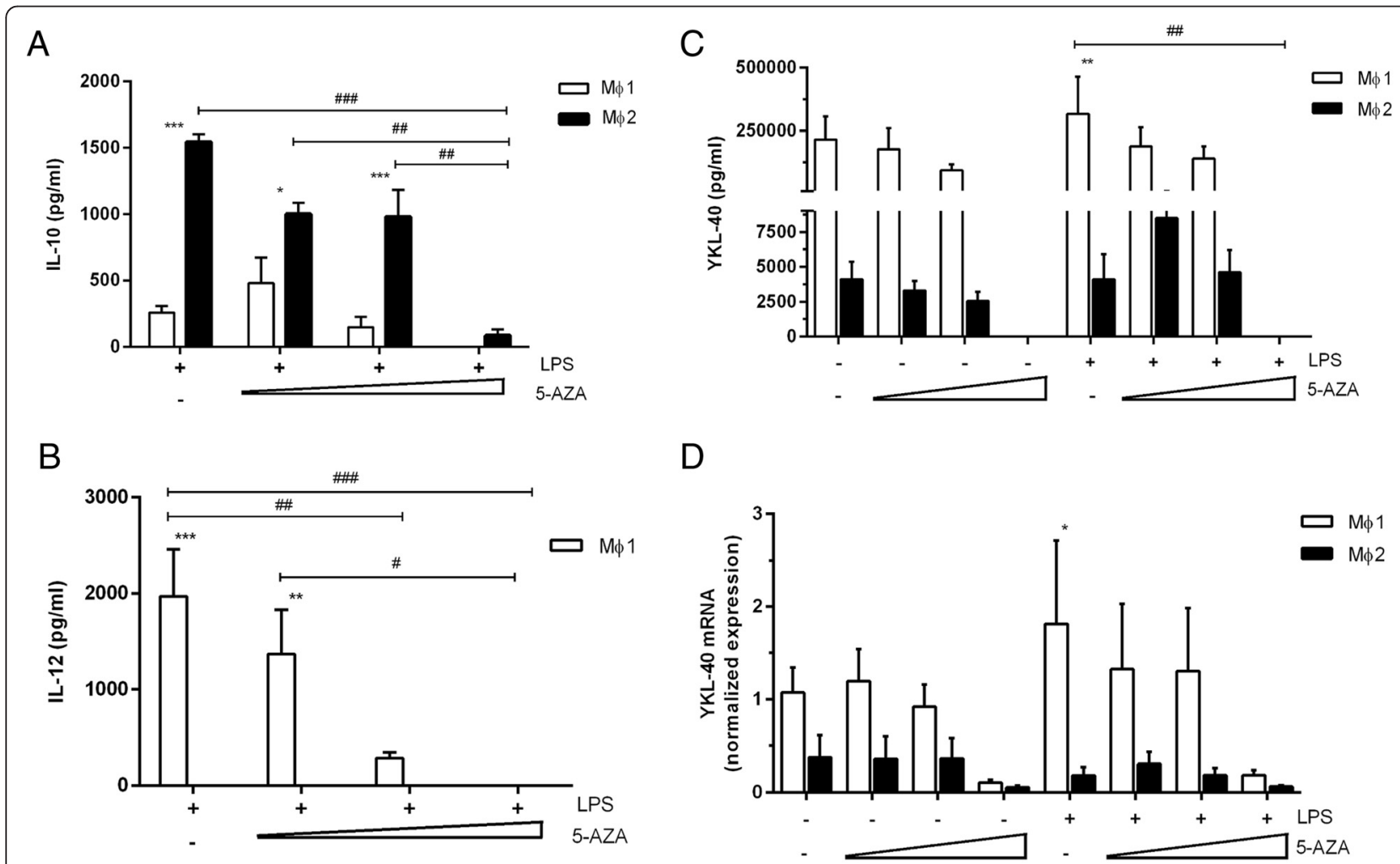

Fig. 5 YKL-40 protein secretion and mRNA expression is inhibited by the demethylating agent 5AZA. IL-10 and IL-12p40 secretion after stimulation with 5-AZA (concentration $0.1,1$ and $10 \mu \mathrm{M}$ ) (a and $\mathbf{b}$, respectively). YKL-40 protein secretion and mRNA expression in MФ1 and MФ2 after culturing with 5-AZA (concentration $0.1,0.3$ and $1 \mathrm{nM}$ ) (c and d respectively). Differentiated cells are stimulated with $100 \mathrm{ng} / \mathrm{ml}$ LPS for $24 \mathrm{~h}$. Data represent mean and SEM ( $n=3$ donors). ${ }^{*} p \leq 0.05,{ }^{* *} p<0.01$ or ${ }^{* * *} p<0.001$ compared to MФ2 with corresponding stimulus. \# $p \leq 0.05$, \#\#: $p<0.01$ or \#\#\# $p<0.001$ compared to corresponding LPS stimulated cells 
Table 2 Patient characteristics of compliant patients at baseline of the GLUCOLD study

\begin{tabular}{lll}
\hline & ICS $(n=51)$ & Placebo $(n=24)$ \\
\hline Gender (M/F) (n) & $45 / 6$ & $20 / 4$ \\
Age (yr) & $61.7(7.8)$ & $59.4(8.1)$ \\
Smoking (y/n) (n) & $33 / 18$ & $17 / 7$ \\
Packyears (yr) & $48(31-56)$ & $42(34-54)$ \\
Post-bronchodilator FEV $1 \%$ pred) & $62.5(9.2)$ & $61.2(8.3)$ \\
Post-bronchodilator FEV 1 (L) & $2.02(0.41)$ & $2.00(0.55)$ \\
Post-bronchodilator FEV $1 /$ IVC (\%) & $47.3(8.8)$ & $46.7(9.0)$ \\
Serum YKL-40 (ng/ml) & $66(49-119)$ & $78(60-118)$ \\
Sputum YKL-40 (ng/ml) & $52(20-79)$ & $18(12-40)$ \\
\hline
\end{tabular}

The ICS group is a combination of the original 30-month fluticasone and 30-month fluticasone with salmeterol groups

Data represent mean with $S D$, median with interquartile range or numbers GLUCOLD Groningen and Leiden Universities Corticosteroids in Obstructive Lung Disease, ICS inhaled corticosteroids, FEV forced expiratory volume in $1 \mathrm{~s}$, IVC inspiratory vital capacity, Pred predicted

\section{Discussion}

This study shows that secretion and expression of YKL40, a chitinase-like protein, is higher in in vitro generated monocyte-derived MФ1 than in MФ2, and that YKL-40 expression is not further increased upon stimulation with several pro-inflammatory stimuli. In addition, YKL-40 release in vitro is strongly inhibited by dexamethasone especially in $M \Phi 1$, most likely due to an effect on differentiation. Addition of the demethylating agent 5-AZA did not significantly decrease YKL-40 release, but did decrease IL12 p40 production by MФ1 and to a smaller extent IL-10 production by MФ2 cells. YKL-40 levels in serum were

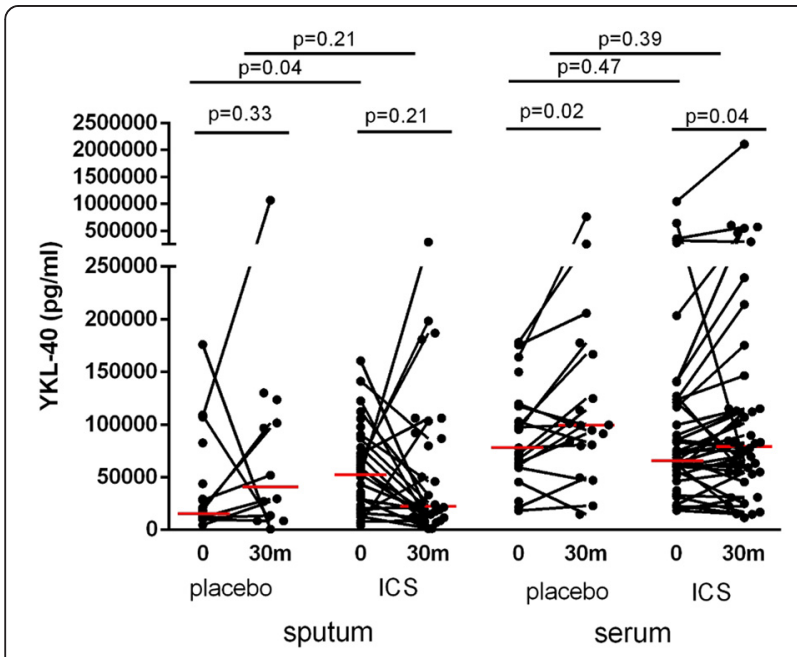

Fig. 6 Sputum and serum YKL-40 protein levels of COPD patients before and after ICS treatment. YKL-40 levels in sputum and serum at baseline (0) and after 30 months $(30 \mathrm{~m}$ ) of inhaled corticosteroids (ICS) and placebo. For comparison of the levels between baseline and 30 months, we only included patients from whom samples were available at both time points (paired data). Each dot represent a single patient, red horizontal bars represent medians significantly higher in serum than in sputum of COPD patients. Treatment of these patients for 2.5 years with inhaled corticosteroids did not significantly change serum and sputum YKL-40 levels compared to placebo. These results suggest that YKL-40 is a promising proinflammatory marker in in vitro cultured pro-inflammatory macrophages, but is less suitable for monitoring in vivo effect of treatment with steroids on YKL-40 in serum and sputum of COPD patients.

We show that YKL-40 is a novel marker of in vitro cultured monocyte-derived MФ1, which is independent of LPS, OSM and TNF $\alpha$. This is an important observation, since many established MФ1 markers require additional stimulation to induce expression. Our data confirm and extend previous results [16, 17, 29], reporting higher CHI3L1 expression in classically activated macrophages compared to alternatively activated macrophages. In the latter studies, in contrast to our study, interferon-gamma (IFN- $\gamma$ ) and IL-4 were used for MФ1 and MФ2 polarization, respectively $[16,17]$. We extended these data by differentiating monocytes with GM-CSF and M-CSF into $M \Phi 1$ and $M \Phi 2$, respectively, and explored the effect of further stimulation after differentiation with several pro-inflammatory stimuli. We found that dexamethasone efficiently suppressed YKL-40 expression and secretion in $M \Phi 1$, but that this was mainly explained by an inhibitory effect of dexamethasone on MФ1differentiation, thus extending previous results [17].

We found that YKL-40 levels in serum were higher than in sputum of COPD patients. Serum YKL-40 levels of our group of patients were comparable with previous studies [13, 30, 31]. However, sputum YKL-40 levels with sputum processed using the whole sample method, were considerably lower than in studies using the selected plug method [12]. This is most likely due to dilution which is inherent to the whole sample method. After long-term treatment with ICS, we did not detect a significant change in YKL-40 levels in serum and sputum compared to placebo. To our knowledge, this is the first study that evaluates the long-term effect of ICS in serum and sputum YKL-40 levels of COPD patients. Therefore, this study presents new in vitro and in vivo data that may help to provide insight in the function of YKL-40.

The mechanisms that regulate YKL-40 expression in health and disease are partly understood. It has been demonstrated that YKL-40 expression is absent in monocytes, and markedly induced during macrophage differentiation, especially during the later stages of differentiation [2]. Promotor analysis of the CHI3L1 gene revealed that especially $\mathrm{Sp} 1$, an ubiquitous transcription factor, is important for CHI3L1 gene expression [6]. Possibly epigenetic mechanisms also contribute to CHI3L1 expression. This is supported by the finding of a SNP 
localized near the CpG island in the promoter region of the CHI3L1 gene, which is associated with YKL-40 expression [19, 20, 32]. Furthermore, hypomethylation of the CHI3L1 gene in rheumatoid arthritis is associated with increased expression of YKL-40 [33, 34]. Therefore, CHI3L1 gene expression may be regulated by transcription factors such as Sp1 and by DNA methylation status. Our observations with 5-AZA treatment do not support a role for DNA methylation in the expression of YKL-40 in MФ1. Further studies into methylation status of the promotor of YKL-40, the role of histone modification and microRNAs are needed to define a role of epigenetic mechanisms in the expression of YKL-40 in (lung) macrophages.

The strength of our study is that it describes a thorough evaluation of a novel, potential pro-inflammatory macrophage marker using both in vitro and in vivo approaches. Well-characterized patients with COPD used long-term, randomized, placebo-controlled treatment with ICS. However, we were unable to detect an effect of a randomized treatment with ICS on YKL-40 serum and sputum levels. Nevertheless, our study has some limitations. First, we used in vitro cultured monocyte-derived macrophages from whole blood of healthy subjects that were differentiated towards $M \Phi 1$ and $M \Phi 2$ instead of lung-derived (e.g. alveolar) macrophages that were differentiated under the influence of the local environment. Since the culture systems do not fully reflect in vitro differentiation of macrophage subsets $[35,36]$, it needs to be noted that the effect of steroids on lung macrophages may differ from that on in vitro differentiated macrophages. Furthermore, in vivo a heterogeneous and intermediate macrophage population exists [37], which complicates the comparison with in vitro generated $M \Phi$ subsets. We therefore cannot formally exclude the possibility that this has contributed to our inability to detect an effect of inhaled corticosteroids on serum and sputum YKL-40 levels. Second, the demethylating agent 5-AZA demonstrated cell toxicity which might have influenced our results. However, we found a dosedependent inhibition of YKL-40 expression and secretion, suggesting that DNA methylation status may contribute to regulation of YKL-40 expression in MФ1, which is in line with studies posing that methylation of a part of the CpG island of the CHI3L1 gene is associated with YKL-40 levels [19, 32].

How can we explain that serum and sputum YKL-40 levels of COPD patients were not significantly changed after long-term ICS treatment compared to placebo? This is unexpected since serum YKL-40 levels of rheumatoid arthritis patients rapidly decreased after one week of prednisolone [18]. However, the amount of inhaled fluticasone that reaches the systemic circulation is low [38], which could explain why serum YKL-40 levels did not significantly change with ICS therapy. In addition, lung macrophages in COPD have reduced glucocorticoid sensitivity $[39,40]$.

\section{Conclusions}

YKL-40 is mainly expressed and secreted by $M \Phi 1$ and is not further increased by pro-inflammatory stimuli. YKL40 release is inhibited by dexamethasone in $M \Phi 1$ in vitro, whereas long-term treatment of COPD patients with inhaled corticosteroids did not significantly change YKL-40 levels in serum and sputum. This suggests that YKL-40 is a potential marker for in vitro cultured pro-inflammatory macrophages and is not a valuable biomarker in serum and sputum of patients with COPD treated with inhaled corticosteroids.

\section{Abbreviations}

5-AZA: 5-AZA-2'-deoxycytidine; BRP-39: Breast regression protein-39; BSA: Bovine serum albumin; COPD: Chronic obstructive pulmonary disease: ELISA: Enzyme-linked immunosorbent assay; FCS: Fetal calf serum; FEV : Forced Expiratory Volume in 1 second; GLUCOLD: Groningen and Leiden Universities Corticosteroids in Obstructive Lung Disease; hCAP: Human cathelicidin antimicrobial peptide; HC gp-39: human cartilage glycoprotein-39; ICS: Inhaled corticosteroids; IL: Interleukin;

LPS: Lipopolysaccharide; OSM: Oncostatin M; SNP:

Single-nucleotide polymorphism; TNFa: Tumour Necrosis Factor-alpha; YKL-40: Chitinase 3-like-1.

\section{Competing interests}

The GLUCOLD study was funded by the Netherlands Organization for Scientific Research (NWO) (grant number 940-35-033), Lung Foundation Netherlands (grant number 93.96.3), GlaxoSmithKline (GSK) of The Netherlands (grant number SC0107656), University Medical Center Groningen and Leiden University Medical Center.

\section{Authors' contributions}

LK, EW, AS and PH designed the study and the experiments. EW was responsible for the cell cultures. LK, EW and AS were involved in ELISA, immunocytochemical staining and GPCR. LK performed the sputum inductions, AS was responsible for processing the serum and sputum samples. LK and EW statistically analyzed the data. DP, HK and PS were responsible for the initiation of the GLUCOLD study and recruitment of the patients. LK, EW and PH drafted the manuscript. All authors have read, revised and approved the submission of the final manuscript.

\section{Financial support}

This study was funded by a grant from the Lung Foundation Netherlands (grant number 3.2.08.0032 and 93.96.3), the Netherlands Organization for Scientific Research (NWO) (grant number 940-35-033), GlaxoSmithKline (GSK) of The Netherlands (grant number SCO107656), University Medical Center Groningen and Leiden University Medical Center.

\section{Author details}

'Department of Pulmonology, Leiden University Medical Center, Albinusdreef 2, NL-2333ZA, Leiden, The Netherlands. ²Department of Pulmonology, University of Groningen, University Medical Center Groningen, Groningen, The Netherlands. ${ }^{3}$ Department of Respiratory Medicine, Academic Medical Center, Amsterdam, The Netherlands.

Received: 29 August 2015 Accepted: 15 December 2015

Published online: 22 December 2015

\section{References}

1. Johansen JS. Studies on serum YKL-40 as a biomarker in diseases with inflammation, tissue remodelling, fibroses and cancer. Dan Med Bull. 2006:53:172-209. 
2. Krause SW, Rehli M, Kreutz M, Schwarzfischer L, Paulauskis JD, Andreesen R. Differential screening identifies genetic markers of monocyte to macrophage maturation. J Leukoc Biol. 1996;60:540-5.

3. Rehli M, Krause SW, Andreesen R. Molecular characterization of the gene for human cartilage gp-39 (CHI3L1), a member of the chitinase protein family and marker for late stages of macrophage differentiation. Genomics. 1997:43:221-5

4. Volck B, Price PA, Johansen JS, Sorensen O, Benfield TL, Nielsen HJ, et al. YKL-40, a mammalian member of the chitinase family, is a matrix protein of specific granules in human neutrophils. Proc Assoc Am Physicians. 1998;110:351-60.

5. Kirkpatrick RB, Emery JG, Connor JR, Dodds R, Lysko PG, Rosenberg M. Induction and expression of human cartilage glycoprotein 39 in rheumatoid inflammatory and peripheral blood monocyte-derived macrophages. Exp Cell Res. 1997;237:46-54.

6. Rehli M, Niller H-H, Ammon C, Langmann S, Schwarzfischer L, Andreesen R, et al. Transcriptional regulation of CHI3L1, a marker gene for late stages of macrophage differentiation. J Biol Chem. 2003;278:44058-67.

7. Johansen JS, Jensen BV, Roslind A, Nielsen D, Price PA. Serum YKL-40, a New prognostic biomarker in cancer patients? Cancer Epidemiol Biomarkers Prev. 2006;15:194-202.

8. Lee CG, Da Silva C, Dela Cruz CS, Ahangari F, Ma B, Kang MJ, et al. Role of chitin, chitinase/chitinase-like proteins in inflammation, tissue remodeling, and injury. Annu Rev Physiol. 2011;73:479-501.

9. Jefri M, Huang Y-N, Huang W-C, Tai C-S, Chen W-L. YKL-40 regulated epithelial-mesenchymal transition and migration/invasion enhancement in non-small cell lung cancer. BMC Cancer. 2015;15:590.

10. Sakazaki Y, Hoshino T, Takei S, Sawada M, Oda H, Takenaka S, et al. Overexpression of Chitinase 3-Like 1/YKL-40 in Lung-Specific IL-18Transgenic Mice, Smokers and COPD. PLoS ONE. 2011;6:e24177.

11. Chupp GL, Lee CG, Jarjour N, Shim YM, Holm CT, He S, et al. A chitinase-like protein in the lung and circulation of patients with severe asthma. N Engl J Med. 2007;357:2016-27.

12. Otsuka K, Matsumoto H, Niimi A, Muro S, Ito I, Takeda T, et al. Sputum YKL-40 levels and pathophysiology of asthma and chronic obstructive pulmonary disease. Respiration. 2012;83:507-19.

13. Letuve S, Kozhich A, Arouche N, Grandsaigne M, Reed J, Dombret MC, et al. YKL-40 is elevated in patients with chronic obstructive pulmonary disease and activates alveolar macrophages. J Immunol. 2008;181:5167-73.

14. Brusselle GG, Joos GF, Bracke KR. New insights into the immunology of chronic obstructive pulmonary disease. Lancet. 2011;378:1015-26.

15. Gordon S, Taylor PR. Monocyte and macrophage heterogeneity. Nat Rev Immunol. 2005;5:953-64

16. Di Rosa M, Malaguarnera G, De Gregorio C, Drago F, Malaguarnera L. Evaluation of CHI3L-1 and CHIT-1 expression in differentiated and polarized macrophages. Inflammation. 2013;36:482-92.

17. Kzhyshkowska J, Mamidi S, Gratchev A, Kremmer E, Schmuttermaier C, Krusell L, et al. Novel stabilin-1 interacting chitinase-like protein (SI-CLP) is up-regulated in alternatively activated macrophages and secreted via lysosomal pathway. Blood. 2006;107:3221-8.

18. Johansen JS, Stoltenberg M, Hansen M, Florescu A, Hørslev-Petersen K, Lorenzen I, et al. Serum YKL-40 concentrations in patients with rheumatoid arthritis: relation to disease activity. Rheumatology. 1999;38:618-26.

19. Ober C, Tan Z, Sun Y, Possick JD, Pan L, Nicolae R, et al. Effect of variation in CHI3L1 on serum YKL-40 level, risk of asthma, and lung function. N Engl J Med. 2008;358:1682-91.

20. Michel S, Busato F, Genuneit J, Pekkanen J, Dalphin JC, Riedler J, et al. Farm exposure and time trends in early childhood may influence DNA methylation in genes related to asthma and allergy. Allergy. 2013;68:355-64.

21. Matsuda A, Asada Y, Takakuwa K, Sugita J, Murakami A, Ebihara N. DNA methylation analysis of human trabecular meshwork cells during Dexamethasone stimulation DNA methylation analysis of trabecular meshwork cells. Invest Ophthalmol Vis Sci. 2015;56:3801-9.

22. Kagoshima M, Wilcke T, Ito K, Tsaprouni L, Barnes PJ, Punchard N, et al. Glucocorticoid-mediated transrepression is regulated by histone acetylation and DNA methylation. Eur J Pharmacol. 2001:429:327-34.

23. Verreck FAW, de Boer T, Langenberg DML, van der Zanden L, Ottenhoff THM. Phenotypic and functional profiling of human proinflammatory type-1 and anti-inflammatory type-2 macrophages in response to microbial antigens and IFN-\{gamma\}- and CD40L-mediated costimulation. J Leukoc Biol. 2006;79:285-93.
24. Wout EFA V't, Van Schadewijk A, Savage NDL, Stolk J, Hiemstra PS. a1-antitrypsin production by proinflammatory and antiinflammatory macrophages and dendritic cells. Am J Respir Cell Mol Biol. 2012;46:607-13.

25. Lapperre TS, Snoeck-Stroband JB, Gosman MM, Jansen DF, Van Schadewijk A, Thiadens HA, et al. Effect of fluticasone with and without salmeterol on pulmonary outcomes in chronic obstructive pulmonary disease: a randomized trial. Ann Intern Med. 2009;151:517-27.

26. Kunz LIZ, Lapperre TS, Snoeck-Stroband JB, Budulac S, Timens W, van Wijngaarden S, et al. Smoking status and anti-inflammatory macrophages in bronchoalveolar lavage and induced sputum in COPD. Respir Res. 2011;12:34.

27. in 't Veen JC, de Gouw HW, Smits HH, Sont JK, Hiemstra PS, Sterk PJ, et al. Repeatability of cellular and soluble markers of inflammation in induced sputum from patients with asthma. Eur Respir J. 1996;9:2441-7.

28. van der Does AM, Beekhuizen H, Ravensbergen B, Vos T, Ottenhoff THM, van Dissel JT, et al. LL-37 directs macrophage differentiation toward macrophages with a proinflammatory signature. J Immunol. 2010;185:1442-9.

29. Hashimoto S, Suzuki T, Dong HY, Yamazaki N, Matsushima K. Serial analysis of gene expression in human monocytes and macrophages. Blood. 1999;94:837-44.

30. Matsuura H, Hartl D, Kang MJ, Cruz CSD, Koller B, Chupp GL, et al. Role of breast regression protein-39 in the pathogenesis of cigarette smoke-induced inflammation and emphysema. Am J Respir Cell Mol Biol. 2011;44:777-86.

31. Holmgaard D, Mygind L, Titlestad I, Madsen H, Pedersen S, Johansen J, et al. Plasma YKL-40 and all-cause mortality in patients with chronic obstructive pulmonary disease. BMC Pulm Med. 2013;13:77.

32. Konradsen JR, James A, Nordlund B, Reinius LE, Söderhäll C, Melén E, et al. The chitinase-like protein YKL-40: a possible biomarker of inflammation and airway remodeling in severe pediatric asthma. J Allergy Clin Immunol. 2013;132:328-35. e325.

33. Nakano K, Boyle DL, Firestein GS. Regulation of DNA methylation in rheumatoid arthritis synoviocytes. J Immunol. 2013;190:1297-303.

34. Nakano K, Whitaker JW, Boyle DL, Wang W, Firestein GS. DNA methylome signature in rheumatoid arthritis. Ann Rheum Dis. 2013;72:110-7.

35. Gordon S, Mantovani A. Diversity and plasticity of mononuclear phagocytes. Eur J Immunol. 2011;41:2470-2.

36. Sica A, Mantovani A. Macrophage plasticity and polarization: in vivo veritas. J Clin Invest. 2012;122:787-95.

37. Hiemstra PS. Altered macrophage function in chronic obstructive pulmonary disease. Ann Am Thorac Soc. 2013;10:S180-5.

38. Falcoz C, Oliver R, McDowall JE, Ventresca P, Bye A, Daley-Yates PT. Bioavailability of orally administered micronised fluticasone propionate. Clin Pharmacokinet. 2000;39 Suppl 1:9-15.

39. Culpitt SV, Rogers DF, Shah P, De Matos C, Russell REK, Donnelly LE, et al. Impaired inhibition by dexamethasone of cytokine release by alveolar macrophages from patients with chronic obstructive pulmonary disease. Am J Respir Crit Care Med. 2003;167:24-31.

40. Ito K, Lim S, Caramori G, Chung KF, Barnes PJ, Adcock IM. Cigarette smoking reduces histone deacetylase 2 expression, enhances cytokine expression, and inhibits glucocorticoid actions in alveolar macrophages. FASEB J. 2001;15:1110-2

\section{Submit your next manuscript to BioMed Central and we will help you at every step:}

- We accept pre-submission inquiries

- Our selector tool helps you to find the most relevant journal

- We provide round the clock customer support

- Convenient online submission

- Thorough peer review

- Inclusion in PubMed and all major indexing services

- Maximum visibility for your research

Submit your manuscript at www.biomedcentral.com/submit
C Biomed Central 